\title{
Research on the experimental road signs perception time and their intelligibility
}

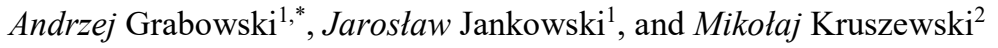 \\ ${ }^{1}$ Central Institute for Labour Protection - National Research Institute, Czerniakowska 16 str., 00-701 \\ Warsaw \\ ${ }^{2}$ Motor Transport Institute, 80 Jagiellońska Str., 03-301 Warsaw
}

\begin{abstract}
Eye tracking technology is often used in road safety research. Eye tracker are used during real and simulated driving to investigate driver's behaviour. It is also used for assessing influence of new elements, like experimental road signs or road signs from different country, on driver's behaviour and performance. However it should be noted that, the probability that the signs will be noticed cannot be solely assessed by eye tracking data. Therefore there is a need to complete the results with additional sources of data, eg questionnaire study. In the paper results of research concerning road signs perception carried out with 33 volunteers are presented. Twelve boards with different road situations in the form of photos taken from the driver's perspective were prepared. After the exposure, the volunteer answered questions about the memorized information presented on the road signs.
\end{abstract}

\section{Introduction and objectives}

Experimental road signs, as new elements observed by drivers can attract attention for too long or too short time in comparison to the other road signs. In the first case this can lead to a dangerous situation, because the attention of the driver for too long will be detached from the movement of other vehicles on the road [1]. In the second case, the effect may be a misunderstanding of the content of the experimental sign and, consequently, making erroneous or inconsistent with the traffic regulations decision.

Studies of traffic sign comprehension in most cases assumes the analysis of sign meaning identification and is carried out using surveys like in the research of Taamneh and Alkheder [2] or Banares et al. [3]. The aspect of dynamic task, that the driving is, is frequently underestimated. New technics for this purpose was proposed in this research, due to lack of objective measures that may be a valuable source of information in dynamic driving condition.

In order to examine cognitive processes of human, the development of eyetracking as a part of cognitive psychology indicated gaze activity as one of the most valuable objective measures [4] driver gazes while driving in a real environment often eye tracking technology is used. Because driver can be distracted by various elements it is important to investigate

\footnotetext{
*Corresponding author: angra@ciop.pl
} 
what factors influences driver attention. Among various visual distractions, objects such as advertisements that are commonly prevailing elements at the roadside, represent an important external distractor that may affect driving performance [5]. The research concerning drivers' perception of roadside elements was performed using eye tracker during ten kilometres of driving in urban area. It was found that drivers' age is not associated with the number of roadside objects detected. Those drivers who detected more traffic signs were also more attentive to visual advertisements [5].

Real images and movie can be also used in simulated environment. In some research a driving simulator mock-up is used and the participants navigate through video from routes. In those videos new traffic signs can be digitally implemented using specialized software for camera-tracking and 3D video-integration [6]. Visual behaviour of the volunteers can be also monitored using eye tracking technology. Because conditions of the experiment is the same for all volunteers, it is possible to get less noisy results.

The eye tracking technology can be also used to evaluate of driver distraction by unfamiliar road signs, eg. road signs from different country [1]. It occurs that participants spent increased time looking at unfamiliar road signs. Misinterpretation occurred due to the influence of previous experience and many drivers drove at reduced speeds throughout to compensate for the anticipated cognitive load [1].

It is also possible to support designing of new road signs with eye tracking technology. In such case the data from eye tracker is used to investigate driver reading patterns. As results of the study show drivers tend to read traffic signs in a top-down, left-right approach. Furthermore, one consistent pattern seen in reading the different traffic signs is that they primarily focus on the center part of the sign, while at the same time having a well-distributed scan path [7].

As any other technology also eye tracking technology has its own limitations, for example drivers may glance at signs, yet fail to identify them and may correctly identify signs in the absence of a glance. Thus, the probability that the signs will be noticed cannot be solely assessed by eye tracking data [8]. Only about half of all signs were recalled just $2 \mathrm{~s}$ after they were passed. About 20 percent of speed limit signs received glances; however drivers were aware of the posted speed limit about 80 percent of the time [8].

The main aim of the conducted research was to determine the time which is devoted to road signs in the context of various traffic situations using eye-tracking [9]. Two experimental road signs were selected: directional marking "E" with numbering of junctions and vertical marking assigned to lanes. Each of these road signs appeared in three different design variants differing in the scope of the presented data (each subsequent variant of the road sign contained more information than the previous one).

\section{Method and results}

The main data source are the results of research carried out with 33 volunteers (16 women and $17 \mathrm{men}$ ) in 3 different age groups: 18-30; $31-50$ and over 50 years old. All of them were active drivers. Twelve boards with different road situations in the form of photos taken from the driver's perspective were prepared for the tests (Fig. 1). On each board, at least one vertical road sign was visible. Exposure to the traffic situation lasted for 3 seconds, during which movement of eyeballs were registered to identify which fragments of road signs the volunteer is looking at (Fig. 2). In each image areas of interests was assigned to road signs (Fig. 3). After the exposure, the volunteer answered questions about the memorized information presented on the signs to determine whether the information contained on the road sign was correctly understood and remembered. 

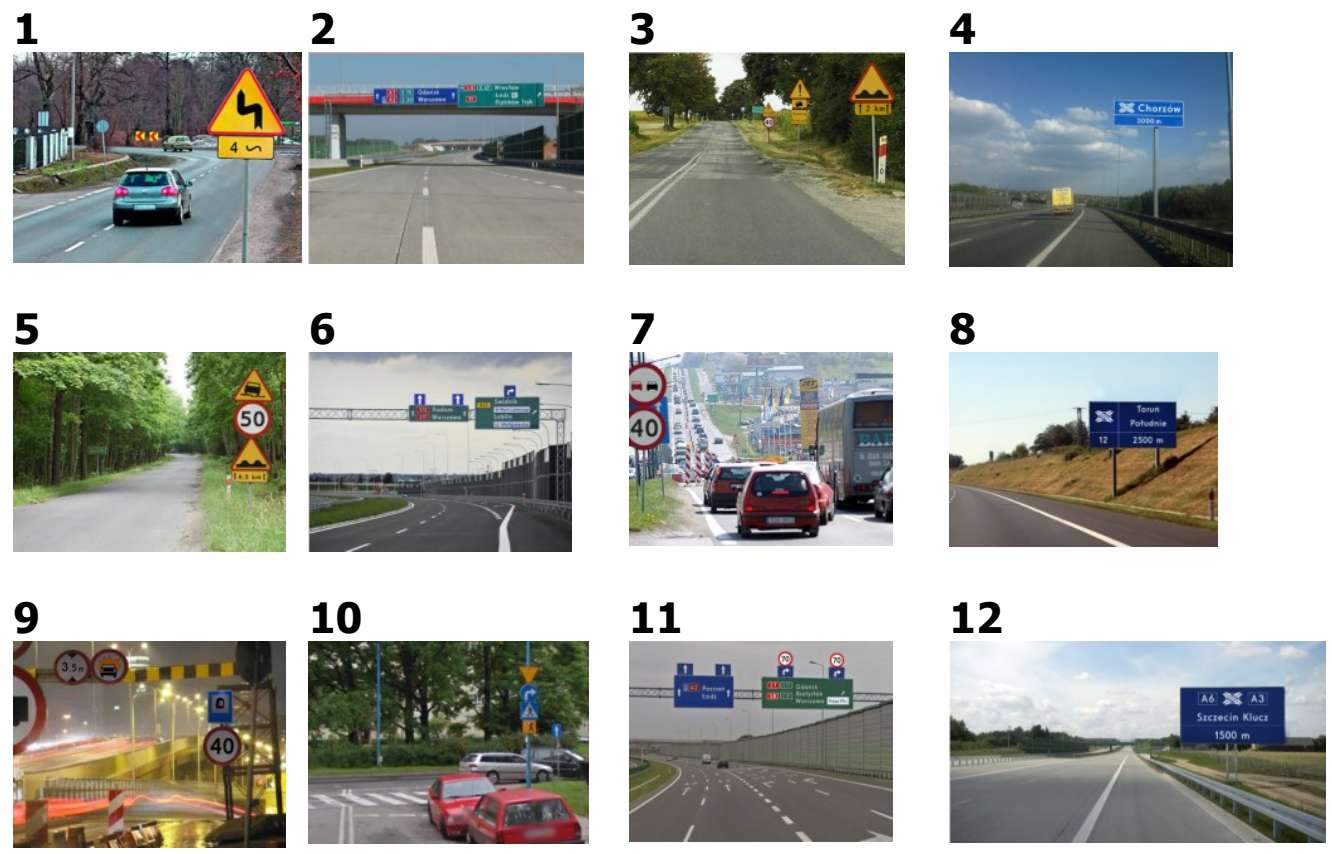

Fig. 1. All road situations.

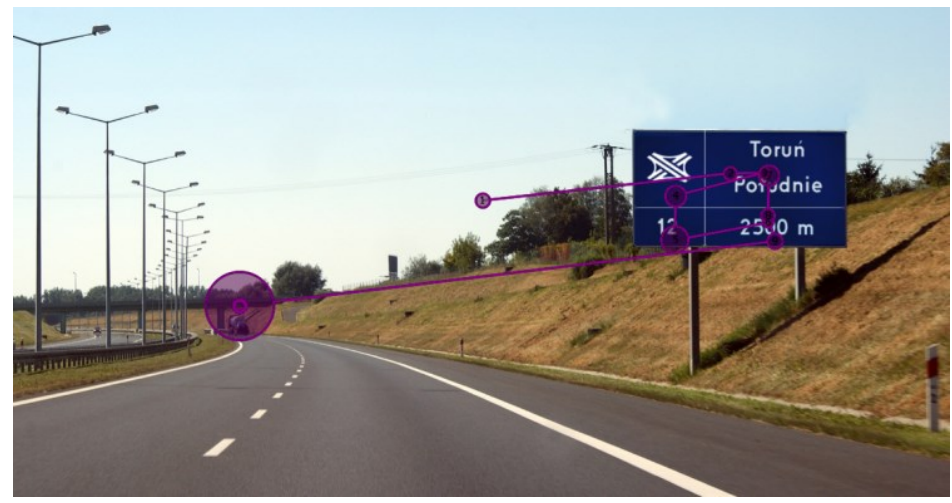

Fig. 2. The focus of sight on the presented picture of the traffic situation was measured. The larger the circle, the longer the focus was on the point. In the presented example, we can see that the volunteer taking part in the study looked the longest at the truck going in front.
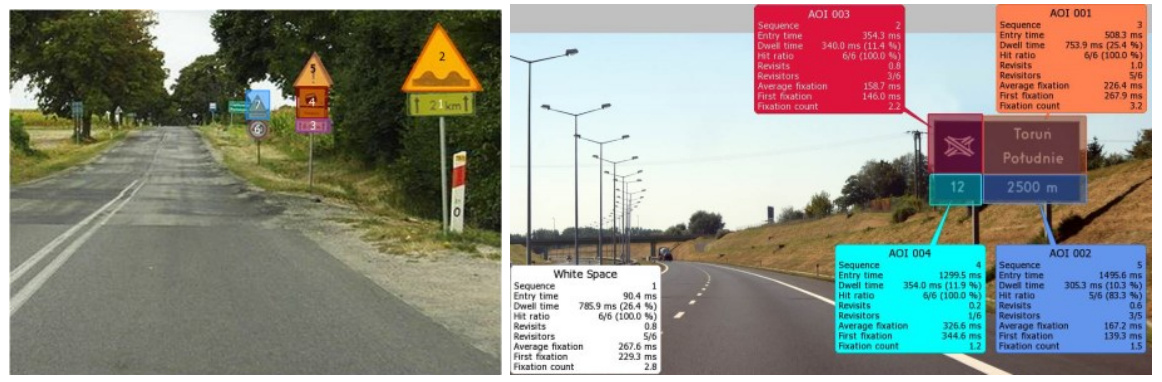

Fig. 3. Examples of areas of interest placed on a picture showing the traffic situation (on the left road situation number 3 is shown). 
Based on the results of the research, time devoted to individual fragments of road signs (Fig. 4 and Fig. 5), the relation between this time and the probability of understanding the content of the road sign and the order of the observed elements of the road environment will be featured. An important research question is also to determine the impact of increasing the amount of information presented by road signs on the ability to understand and remember this information.

One of the main parameters that can be quantified with the use of an eye tracker is the time of eye focus, and so also the attention, on the sign or its fragment. In this context, it is important to compare the experimental signs with the signs used so far, and thus those to which the drivers are already accustomed. It turns out that the time of focusing on the experimental characters regarding the question is much larger and these differences are statistically significant. The typical sign attracted attention on average to $567 \mathrm{~ms}$, while the element of the experimental sign was $781 \mathrm{~ms}$. The observed increase in this time is almost $40 \%$. Similar values are also observed for the group of questions for which a correct answer was given and a group of questions for which an incorrect answer was given. In both cases, the observed differences are statistically significant, and the numerical values are very similar (respectively $579 \mathrm{~ms}$ and $788 \mathrm{~ms}, 534 \mathrm{~ms}$ and $757 \mathrm{~ms}$ ). Similarly for individual age groups. The observed differences are statistically significant, and the numerical values are very similar (eg the average eye focus time on the experimental sign is $772 \mathrm{~ms}, 787 \mathrm{~ms}$ and $781 \mathrm{~ms}$ for subsequent age groups).

Significantly, differences were also observed in the group of the youngest drivers (18-30 years) and the time of eye focus on the mark depending on the type of response. The time of eye focus on the sign was longer in the case of normal responses (588 ms and $716 \mathrm{~ms}$ respectively). With separate treatment of standard and experimental characters, statistically significant differences were observed only in the case of men. For the whole population, statistically significant differences in the time of observation of the mark depending on the type of response were observed only for traffic situations: 1,3 and 11 .

Sign observation time is not the only important factor. It turns out that the people who gave the wrong answer started looking at the question mark noticeably later (on average in 944 milliseconds versus 798 milliseconds for correct answers). The observed difference is also statistically significant for standard characters, while for experimental characters statistically significant differences are observed only for the 31-50 age group (this is on average 1125 milliseconds and 815 milliseconds for abnormal and correct answers). For individual traffic situations, statistically significant differences were observed only for road situations 3, 10 and 11 (see Fig. 1).

The next analysed factor was the order in which the viewer started to look at a sign or a question element. Usually the volunteer taking part in the study focused his attention on the following signs, returning to the previously observed time. It is therefore possible to calculate such changes in the area of concentration before the first look at the question mark. It turns out that the division into standard and experimental characters is strongly related to the type of response. However, for correct answers, this relationship is the reverse of the incorrect answer. In the first case, volunteers focused their eyesight later on experimental characters (the average experimental mark regarding the question was 6.2 in order of looking, while the standard mark 5.0), while in the second it was earlier ( 8.8 for standard and 6.6 for experimental). The numerical values for experimental characters are similar, suggesting differences in the perception of standard characters. The better known signs, i.e. those whose meaning is well known, attract attention much earlier. This is confirmed by tests comparing the order of looking for incorrect and correct answers. The average values obtained for the abnormal responses are noticeably higher and statistically significant for all characters and standard characters (this relationship is not statistically significant for experimental characters, which by definition should be poorly known by all participants of the study). Statistically significant differences are observed for standard characters in all age groups and for traffic situation no. 1 and 11. 
An important factor affecting road safety is the time needed to interpret the mark. In the conducted study, the influence of various values on this time can be estimated based on the measurement of the response time. It turns out that the response time is significantly longer for standard characters, on average $4618 \mathrm{~ms}$ relative to $4026 \mathrm{~ms}$ for experimental characters. A similar relation is also observed in the case of correct answers (4368 ms for standard characters and 3745 for experimental characters). This is related to the fact that the interpretation of less well-known images requires more resources, including more time. This is clearly visible when comparing correct and incorrect response times (3,993 $\mathrm{ms}$ and $5072 \mathrm{~ms}$ respectively). The invalid response time is over $25 \%$ longer. The relative increase in the response time is greater for experimental characters $(33 \%)$ than for standard characters $(23 \%)$. A statistically significant longer time of abnormal responses is observed in all age groups and for traffic situations No. 1, 5, 6, 7, 8, 9 and 12.

The time of answering the question is also dependent on the age group. It is clearly larger for the age groups of older people, eg the average response time for all questions is 3,972 $\mathrm{ms}$ for people aged 18-30, while for people 50+ it is already $4573 \mathrm{~ms}$.

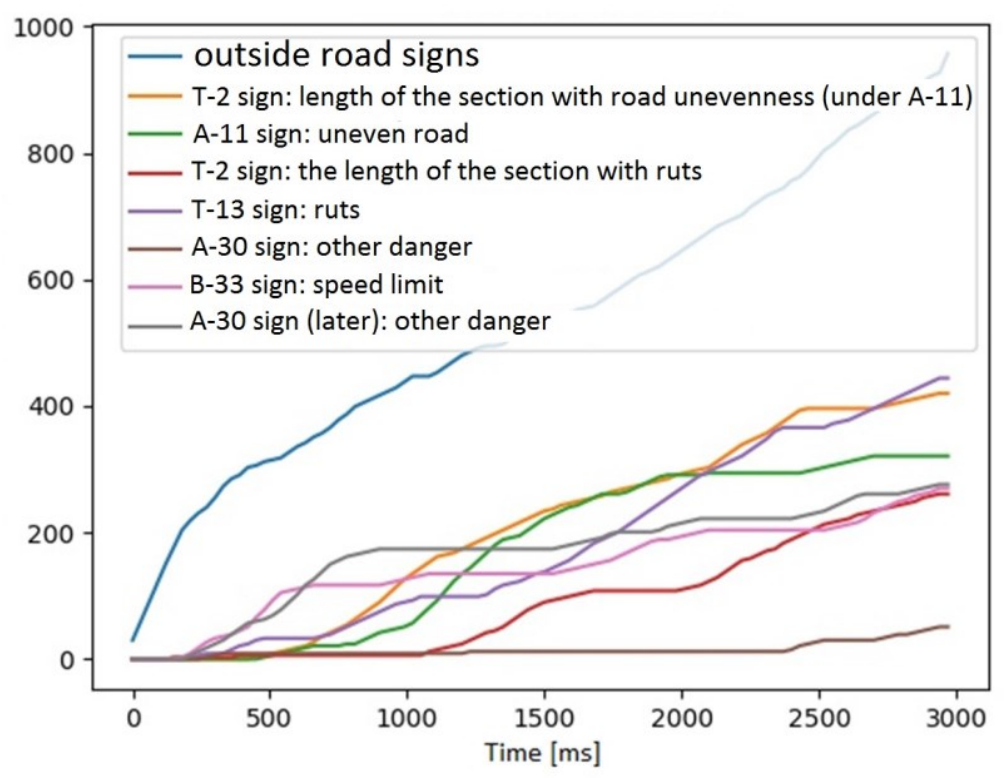

Fig. 4. Aggregated results regarding the time of eye focus on road signs for people from the 18-30 age group and road situation no. 3 . 

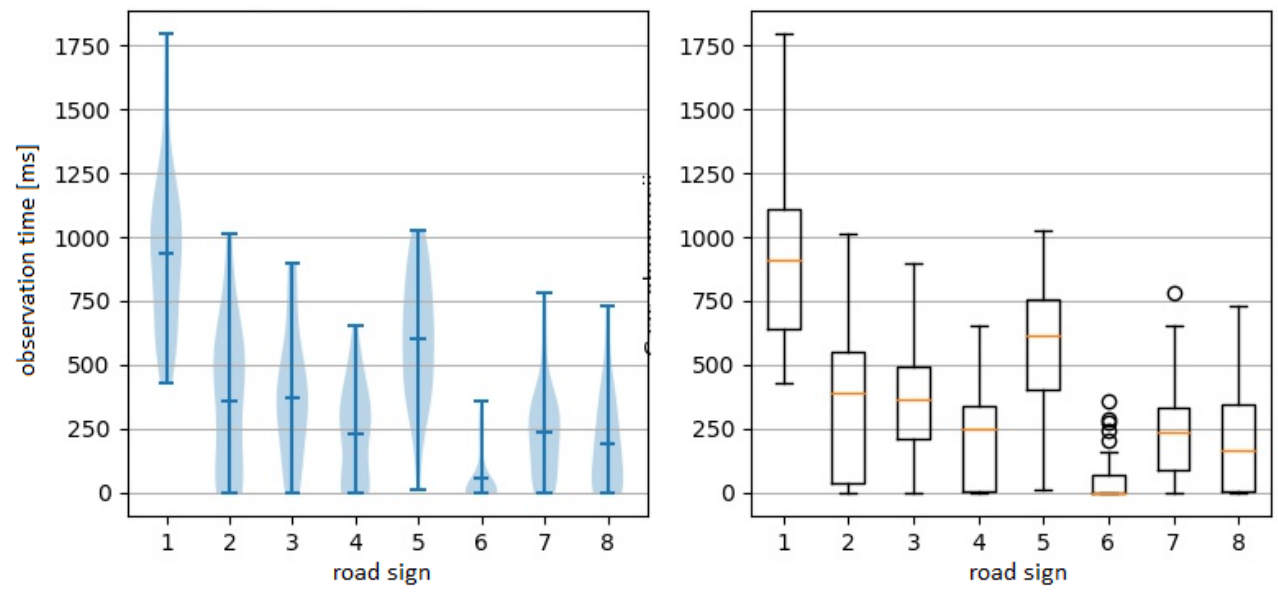

Fig. 5. Time of observation of individual signs for traffic situation 3. Character description: 1. Beyond the signs. 2. T-2 mark The length of the section with road inequalities (under A-11). 3. Mark A-11 Uneven road. 4. T-2 mark The length of the section with ruts. 5. The T-13 mark of the Rool. 6. A-30 sign Other danger. 7. Sign B-33. Speed limitation. 8. A-30 mark (further) Other danger.

\section{Conclusions}

The results of research carried out with eye-tracking are a valuable source of information on the perception of road signs, especially that it is possible to present real, not synthetic, as in the case of simulators, photographs depicting various types of road situations from the driver's perspective.

The main advantage of using the eye tracking is the possibility of objective assessment of the concentration of the driver's attention on selected elements of the picture presenting the selected traffic situation. This allows you to determine the attention span of the selected area with an accuracy of several milliseconds. The test consists in presenting a series of images (slides) with traffic situations. Before the test, the eye must be calibrated, however, due to the visual impairment, some people will not be able to take part in the study. This is particularly problematic in the case of older people, whose vision defects are much more common. Therefore, the results of older people (eg 50+) should be approached with a greater reserve, because they can be artificially inflated by the selection of people who meet the conditions that enable the correct calibration of the eye tracker.

Like any measuring device, the eye tracker has its limitations. First of all, it is generally not possible to correctly monitor the focus of sight all the time due to the limitations of the camera observing the movement of the eyes and the effectiveness of the machine vision algorithms. Values over $90 \%$ should be considered very good and over $80 \%$ as sufficient. It should also be remembered that the eye tracker allows you to determine the focal point of sight, while the person has the ability to correctly perceive images beyond this point, and even that are relatively far away. Therefore, the results of measuring eye focus are insufficient for full assessment, it is suggested to verify understanding and remembering the information presented by means of a few questions (in addition, the mere fact that a volunteer participating in the study looked at a given sign can not indicate that he understood the content of this sign).

Tests with eye tracker may help at the design stage, supporting the process of creation, in classic designing process, but also using ie. design thinking method [10]. Objective eyetracking measures may eliminate the experimental road signs at the design stage, which may lead to one of the two undesirable effects. First, it may pose a threat to road safety by 
excessive attention and concentration of the driver (this time the eye tracker allows to measure, which gives a chance to compare the values obtained to other signs visible in a given situation road traffic).Second, experimental road signs, which probably will not fulfill its role because the attention of drivers does not attract at all or the drivers focus their eyes on it for a very short time preventing correct interpretation and remembering the presented content. Eyetracking in this context may be a valuable form of verification of the sign design.

The work was conducted under the Road Innovations Development program (RID) organized and funded by the National Centre for Research and Development and the General Directorate for National Roads and Motorways (contract No. DZP/RID-I-36/5/NCBR/2016).

\section{References}

1. S. Hurtado, S. Chiasson, An Eye-tracking Evaluation of Driver Distraction and Unfamiliar Road Signs, in: Proc. 8th Int. Conf. Automot. User Interfaces Interact. Veh. Appl. - Automotive’UI 16, pp. 153-160 (ACM Press, New York, USA, 2016). doi:10.1145/3003715.3005407

2. M. Taamneh, S. Alkheder, Traffic sign perception among Jordanian drivers: An evaluation study, Transp. Policy. 66 pp. 17-29 (2018). doi:10.1016/J.TRANPOL.2018.02.017

3. J.R. Bañares, S.A. Caballes, M.J. Serdan, A.T. Liggayu, M.F. Bongo, A comprehension-based ergonomic redesign of Philippine road warning signs, Int. J. Ind. Ergon. 65 pp. 17-25 (2018). doi:10.1016/J.ERGON.2018.01.011

4. M.K. Eckstein, B. Guerra-Carrillo, A.T. Miller Singley, S.A. Bunge, Beyond eye gaze: What else can eyetracking reveal about cognition and cognitive development?, Dev. Cogn. Neurosci. 25 pp. 69-91 (2017). doi:10.1016/j.dcn.2016.11.001

5. D. Topolšek, I. Areh, T. Cvahte, Examination of driver detection of roadside traffic signs and advertisements using eye tracking, Transp. Res. Part F Traffic Psychol. Behav. 43 pp. 212-224 (2016). doi:10.1016/J.TRF.2016.10.002

6. T. De Ceunynck, C. Ariën, K. Brijs, T. Brijs, K. Van Vlierden, J. Kuppens, M. Van Der Linden, G. Wets, Proactive Evaluation of Traffic Signs Using a Traffic Sign Simulator, Eur. J. Transp. Infrastruct. Res. 15 pp. 184-204 (2015)

7. J. Chan, P. Gonzalez, E.M.M. Perez, Designing Traffic Signs: A Case Study on Driver Reading Patterns and Behavior, in: 16th Philipp. Comput. Sci. Congr. Conf., (Puerto Princesa, Palawan, Philippines, 2016)

8. V.W. Inman, Conspicuity of Traffic Signs Assessed by Eye Tracking and Immediate Recall, Proc. Hum. Factors Ergon. Soc. Annu. Meet. 56 pp. 2251-2255 (2012). doi:10.1177/1071181312561474

9. T. Kamiński, M. Kruszewski, M. Niezgoda, P. Soluch, Application of eyetracking research techniques into road safety studies - theoretical considerations, J. KONES. 19 pp. 259-266 (2012)

10. M. Kostrzewski, One Design Issue - Many Solutions. Different Perspectives of Design Thinking - Case Study, in: Uden L., Hadzima B., Ting IH. Knowl. Manag. Organ. KMO 2018. Commun. Comput. Inf. Sci., (Springer, Cham, 2018). doi:10.1007/978-3-319-95204-8_16 